\title{
Colour spaces - a review of historic and modern colour models*
}

\section{GD Hastings and A Rubin}

\author{
Department of Optometry, University of Johannesburg, PO Box 524, Auckland Park, 2006 South \\ Africa
}

$<$ gdhastings1@gmail.com>

$<$ arubin@uj.ac.za>

Received 12 April 2012; revised version accepted 31 August 2012

\begin{abstract}
Although colour is one of the most interesting and integral parts of vision, most models and methods of colourimetry (the measurement of colour) available to describe and quantify colour have been developed outside of optometry. This article presents a summary of some of the most popular
\end{abstract}

colour models and a brief history of the advancements that have led to our current understanding of the complicated phenomenon of colour. ( $S \mathrm{Afr}$ Optom 2012 71(3) 133-143)

Key words: Colour space, colour model, colourimetry, colour discrimination.

\section{Introduction}

The description and quantification of colour, and the even more complicated matter of colour perception, have interested philosophers and scientists for over 2500 years, since at least the times of the ancient Greeks ${ }^{1}$. Colour is a fundamental part of the sense of sight and has a fascinating effect on how people perceive the world - the poet Julian Grenfell even went so far as to say that "Life is Colour"2.

Although the profession of optometry is generally familiar with the defects and deficiencies of colour vision and the tests available to detect such problems, many of the greatest advancements in the understanding, quantification, and reproduction of colour have come from developments elsewhere, in fields such as physics, engineering and psychology. Much of the impetus for these developments came from the printing, textiles, and computer graphics industries because as these industries became more sophisticated, more universal, and more commercial, models and systems needed to be evolved to allow an increasingly more precise and comprehensive description of colour and colour perception.

Some of the earliest attempts at representing colour seem to have been inspired by the change of night to day and involve a linear (one-dimensional) colour scale ranging from black to white with all possible colours in between ${ }^{3}$. An example is Athanasius Kircher's 1646 five-member colour scale, which illustrated a change from black, through blue, red, and yellow, to white. (This model was adapted from the similar 1613 model of Francois d'Aguilons ${ }^{3}$.) Later, radiometry showed that different wavelengths of light could produce different perceptions of colour and thus established an association of certain colours with specific

*This paper is based on research for the postgraduate degree of the first author with the supervision of Professor A Rubin of the University of Johannesburg 
wavelengths. It quickly became apparent, however, that the experience of colour perception could not be quantified fully by using the specification of wavelength alone ${ }^{1,4}$. Thus the subjective nature of colour perception gradually became acknowledged and accepted, and more advanced colour models such as Newton's circle evolved that, for apparently the first time, began regarding colour as a two-dimensional (2-D) quantity 1,3 .

The idea of colour being a multivariate quality was taken further when Mariotte suggested in 1717 that three primary colours could produce any desired colour when used in the proper combination ${ }^{1}$, and the first known system that regarded colour as a threedimensional (3-D) element was the 1758 double-triangle-pyramid proposed by Tobias Mayer ${ }^{3}$. Although many subsequent 3-D theories became popular in the 18 th and 19th centuries, most were neither accurate nor realistic (however, some such as Maxwell's triangle, which will be elaborated on later, formed the foundation of our current understanding of colour) and before further improvements to the modeling and representation of colour could be made, a better understanding of the perceptive processes involved needed to be realised.

Two of the currently best-known and fundamental colour theories that emerged, propose different ways that colour perception could result from three components or processes. More detail on these theories can be found in references 1 , and 5 to 11 ; only a brief overview of each is included here.

The first theory is known as the trichromatic theory. Young, Helmholtz, and Maxwell contributed significantly to formalizing this theory, which proposes the existence of three sets of sensory mechanisms (cone cells) whose unique and independent sensitivity provide a basis for colour discrimination ${ }^{5,6}$. Trichromatic theory incorporated the idea of colour-mixing (when two or more colours coincide spatially and temporally so as to be seen as a single colour) and proposed that, similar to the idea of the Maxwell colour-triangle (a triangle whose three corners represent the three primary colours being mixed (commonly, spectrally pure red, green, and blue) and whose sides enclose the range, or gamut, of all possible combinations of those three primaries), all colours can be formed by the mixing of three primary colours ${ }^{7}$. This theory, therefore, formalized Mariotte's 1717 suggestion.

The sensitivities of the three types of receptor cells were quite specifically determined through separate colour-matching experiments early in the 20th century by Wright and Guild ${ }^{1,12}$. Although some of the assumptions made during those experiments have been questioned, the spectral sensitivity functions that were determined (for an average eye) from those empirical data have remained an accepted and standard part of colourimetry 9,10 . Linear transformations of the three functions (involving short-, medium-, and long-wavelengths respectively) are known as colour matching functions and have formed the basis of some of the most popular colour systems to date.

The second theory, namely Hering's opponentpairs theory, proposes that colour perception results from three complex and antagonistic processes each involving a pair of opposing colour sensations. The three pairs are defined as black-white, red-green, and yellow-blue. A specific colour is thought to be perceived only when its stimulation of the visual system is greater than that of its paired colour (otherwise, when the two colours are equally balanced an achromatic grey is perceived $)^{1,5}$. This theory also incorporates the three colour-matching functions mentioned above, by defining one function as the luminance (brightness) of the perceived colour (thus relating to the black-white process), and the other two functions as representing the chromatic content of the colour ${ }^{3}$.

Each theory has, however, subsequently been found inadequate to fully describe human colour perception on its own. Even from the time of Maxwell's work it could be shown that not all real colours could be produced from a combination of three real primaries as the trichromatic theory suggests ${ }^{5,7}$. And, although Hurvich and Jameson ${ }^{13}$ gave the opponent-pairs theory some mathematical rationalization, it is founded upon largely subjective processes and is difficult to experiment on objectively. Thus, more recently it has been suggested that each theory could be valid and applicable to a separate part in the complex overall process of colour perception 5,11 .

Despite that the exact neural mechanisms and processes involved are still not yet fully understood, the most popular systems of colourimetry use three variables to specify colour. But, subsequent to the abovementioned theories, a more accurate requirement of a 
colour space was realised and better models could be evolved such as those that will be discussed below.

\section{Colour Spaces}

\section{A Colour Space, Defined}

A colour space serves as a means of uniquely specifying, creating, and visualizing colours ${ }^{4}, 14$. Because the process of colour perception is a subjective and inherently variable process, colour spaces aim to describe and standardize colours either between different people, or for machines, or for both ${ }^{4}$. Colour spaces have been divided into two broad groups, namely psychological spaces and geometric spaces. While the former are constructed based on experimental data from subjects (with normal colour vision) and arrange colours according to how they are perceived (with the distance between colours in the space attempting to approximate their observed difference), the latter is a mathematical layout of colours, arranged according to a measured property, defined within a specified range $3,4,15$. Some early colour spaces, such as the Munsell colour space, were purely collections of colours chosen and arranged according to how they were perceived. However, most of the colour spaces discussed in this article began mathematically and were especially modeled to agree more closely with human colour perception $1,3,4$. The more closely the distance or separation between colours in a colour space agrees with the human perception of the magnitude of the perceived difference between them, the more that colour space is said to be visually uniform $^{3,5,8}$.

The three parameters or co-ordinates, which describe the position of a colour within a 3-D space usually do not describe the appearance of the colour, and only have meaning within the context of the specific colour space being used. (This is because of the many other factors, such as ambient lighting, texture of the surface, and surrounding colours, which influence the appearance of a colour.) Similarly, a colour space and all of its colours are only expressible in the units (or scale steps) that make up the space ${ }^{3,4}$. Although some colour-appearance-spaces have been developed (such as the CIECAM97s and the CIECAM02 models ${ }^{16}$ ) that could describe the appearance of a colour, they take into account such a large number of factors and variables (for instance, colourmetric values of: the image, the illuminant, a reference white, and areas of the surrounding field), and are so cumbersome to use, that they have not yet achieved popularity in any industry 4 and will not be included in this article.

Any colour point within a colour space can, therefore, be specified either by its co-ordinates (specific to that colour space) or by a vector that similarly illustrates the position of the colour point relative to a reference point within the space (such as either the origin of the space or another reference colour within the space) ${ }^{14,17}$. Mathematical operations such as addition and subtraction are defined within colour spaces but because some of the colour spaces discussed below are not true vector or mathematical spaces, interesting complications can occur near or across the boundaries or limits of the spaces concerned. These issues have important implications regarding statistical and other mathematical analyses that sometimes involve such boundary colours or situations.

Some of the historically and currently most popular, influential, and useful colour spaces will be discussed in the following sections.

\section{CIE Colour Spaces}

Presently, the most popular system of colour representation is the Commission International d'Eclairage (CIE) (or, in English, the International Commission on Illumination) system, first published in 1931, which has become the international platform to standardize and reproduce colours across many different professions and industries and is considered to be the basis of most modern colourimetry 1, 4, 5, 7. The CIE model is designed around an altered Maxwell triangle that illustrates the proportions of each primary colour rather than the actual amount used, which is converted into rectangular co-ordinates (because the three proportions always sum to one (unitary), only two co-ordinates need be specified and the third can be calculated). In this representation of tristimulus vectors only information regarding the intensity of the colour is lost, while all of the information about the proportional make-up or composition is retained. Such a plot or plane of normalized colours is called a chromaticity diagram, and the co-ordinates of the normalized vectors are called chromaticity co-ordinates ${ }^{1,5,7,18}$.

A curved line of spectral (fully saturated) colours 
enclosing the gamut of all real colours (by the addition of a non-spectral line of purples) is superimposed onto this triangle resulting in a horse-shoe-shaped diagram with white at the approximate centre, and non-spectral colours filling the space, each increasing in saturation away from white towards the line of spectral colours at the diagram's boundary. It was found, however, that many colours that could be mixed from the red, green, and blue primaries fell outside that gamut (examples of the shortcoming of the trichomatic theory mentioned above) and that a primary needed to be added to the sample colour in order to match it with the combination of the other two (it was therefore considered a negative quantity in the proportion-mix ${ }^{1,5,7,18}$ ). To avoid these negative specifications the underlying triangle was enlarged to include the full curve of all real mixed colours.

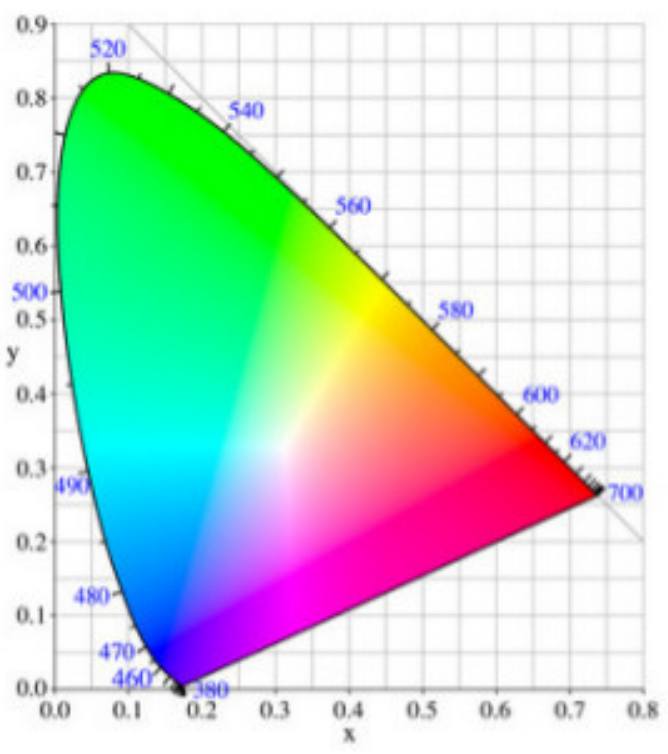

Figure 1: The 1931 CIE chromaticity diagram. The coloured region or gamut forms a 2-D colour projection plane, or crosssection, perpendicular to the luminance axis through the whole 3-D colour-solid of normal colour vision.

Although this resulted in all proportions of the primaries being positive it meant that, now, the three primaries being mixed fell outside the gamut of real (possible) colours and became purely theoretical primaries. They became known as $X, Y$, and $Z$ when referring to the actual amount of each primary, and $x, y$, and $z$ when referring to their relative proportions of a colour make-up (and as mentioned, $x+y+z=1$ ).

$X, Y$, and $Z$ for a sample colour are calculated by multiplying the spectrophotometric properties (wave- length) of the sample with the colour matching functions mentioned above. Because many selections could be made for these three theoretical primaries the CIE chose the properties of the $Y$ primary (the colour matching function of $Y$ ) to correspond with the standard luminosity curve of the eye, and therefore to represent the brightness, or luminance, of the colour being described $1,4,5,7,18$. By specifying two colour proportions ( $x$ and $y$ ) as well as the quantity $Y$ the CIE system enables a user to accurately reproduce a colour (provided illumination is the same).

Despite the usefulness and popularity of the CIE 1931 model, it has flaws such as: that the colours are not uniformly represented on the diagram, and that an especially large proportion of the diagram is allocated to the various green colours ${ }^{5,8}$. The first major improvement on the 1931 model was the 1960 CIE Luv diagram, which attempted an approximately uniform colour space. This space is a linear transformation of the 1931 CIE diagram where colours are similarly represented by specifying the luminance, $L$ (which is unchanged from $Y$ in the 1931 diagram), and two normalized proportions, $u$ and $v$, but the distance between colour points on the diagram approximates the perceived difference between those colours more closely $4,6,10,17$. However, the CIE Luv diagram is considered to compress all yellow, brown, orange, and red colours into a disproportionately small area, which are particularly important colours to the textile and printing industries ${ }^{4,10,17}$.

The 1960 model was further developed via a non-linear transformation into the 1976 CIE Lu'v' system, which shows a further improvement in the correlation of Euclidian distances (the direct and measurable distance between two points, given in Cartesian space by the Pythagorean-triangle formula) between colours on the diagram and the perceived colour differences by an observer. The areas of the 1976 CIE Lu'v' diagram allocated to each colour group are also considered much more balanced (as illustrated in Figure 2). However, this technically more sophisticated model has not replaced its inferior predecessor, as most industries still make use of the 1931 system $^{4}, 6,10,19,20$. (The abovementioned models illustrate that chromaticity diagrams can be constructed in many ways - depending on the choice of primaries ${ }^{21}$.) 


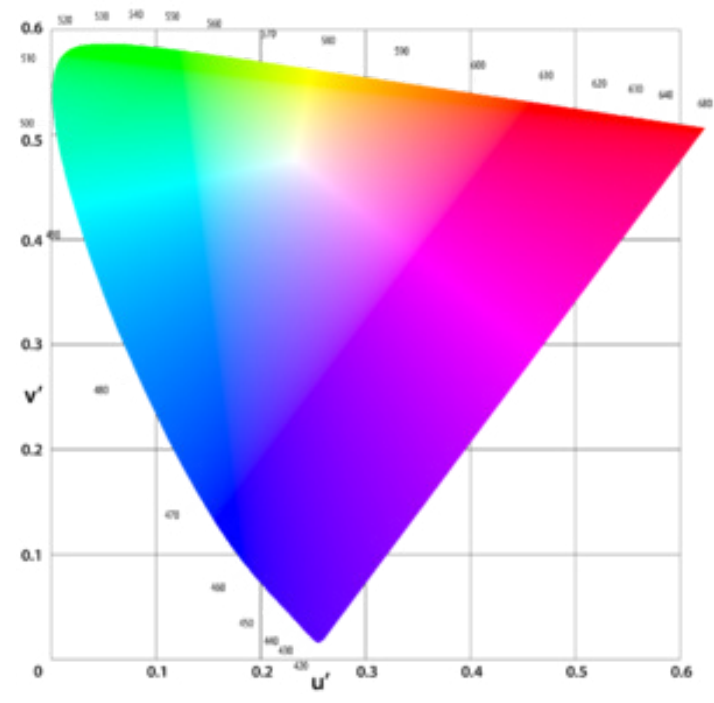

Figure 2: The CIE 1976 chromaticity diagram, shows a more appropriate allocation of area to each colour, as discussed in the text.

Despite being based on three-dimensional theories, all of the above models are, in fact, still merely twodimensional representations of colour (the third variable can be calculated, but is never visualized in the model). Ironically, this is the reason for the popularity of chromaticity diagrams - it is much easier to draw and visualize in 2-D than in 3-D.

Also in 1976, the CIE attempted a truly 3-D representation of colour, the CIE Lab colour space. CIE $L a b$ is derived by another non-linear transformation directly from the 1931 model, and organizes colours around a set of three perpendicular axes: a vertical ' $L$ ' axis that relates to the luminance of the colour and ranges from white to black, and two horizontal axes ' $a$ ' and ' $b$ ' which range from red to green and from yellow to blue respectively. By definition, red and yellow are on the positive side of their respective axes, while green and blue are on the negative sides. Black is positioned at the origin (zero-point) of the vertical axis, while pure white is considered at its positive limit (one hundred). When plotted on these axes, the human colour gamut forms an irregular cone-shaped solid with its apex at the origin. Figure 3 illustrates the effect of lighting on the perception of colour as the visible gamut tapers dramatically at low luminance levels, and also reduces in size at very high luminance levels. ${ }^{4,}$ 19, 22, 23.

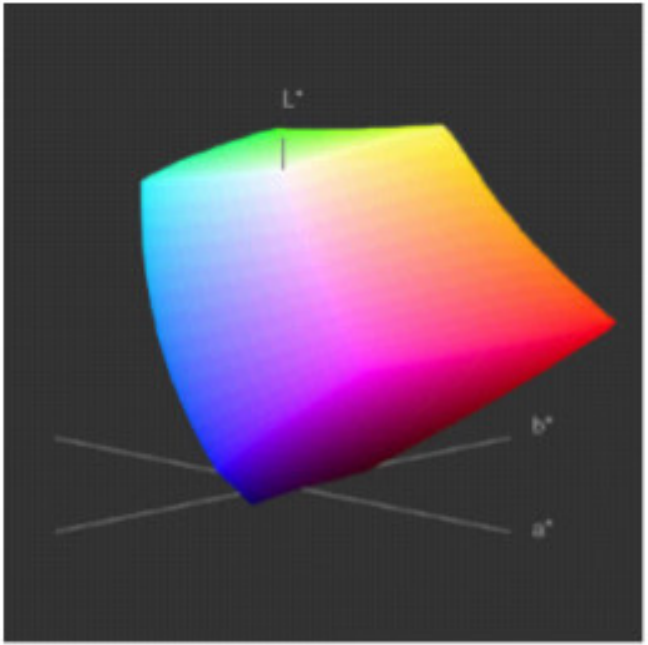

Figure 3: A visualization of the three-dimensional CIE Lab colour space. (Courtesy of G. Hoffmann ${ }^{22}$ ). Note how the perceivable colour gamut decreases at very high luminance $\left(\mathrm{L}^{*}\right)$ levels and especially at low luminance levels.

The CIE $L a b$ system is considered to be one of the most accurate in its organization of colours, resulting in numeric distances between colours agreeing even more accurately with the observed difference between those colours than for that of any of the preceding models ${ }^{6,22,23}$. The CIE Lab model is, however, difficult to reproduce and visualize properly in conventional media and many different perspective-views need to be given.

\section{RGB Colour Space}

Another colour space that has hitherto been difficult to visualize is that which exists for colour television screens and computer monitors. Although modern technology is changing rapidly, these products usually involve the proportional mixing of three monochromatic spectra of light to create a colour (according to the principles of trichromacy mentioned above). The space gets its name from the three spectra that are mixed, namely Red, Green, and Blue (and because it is formed directly from colour-mixing, it is known as an additive colour space) $)^{4,24,25}$. Most cathode ray tube (CRT) monitors and plasma screens are manufactured using the same set of three phosphors (a version of phosphorus P22) to create the three respective spectra and therefore all create a very similar triangular gamut of colours when visualized on a chromaticity diagram, such as the $1931 \mathrm{CIE}$ model $^{26}$. 
Although this gamut falls well within the limits of the full range of visible colours (our perceptual space) (especially in the green part of the spectrum), as can be seen in Figure 4, it is thoroughly sufficient to display images realistically14, 19, 27, 28 .

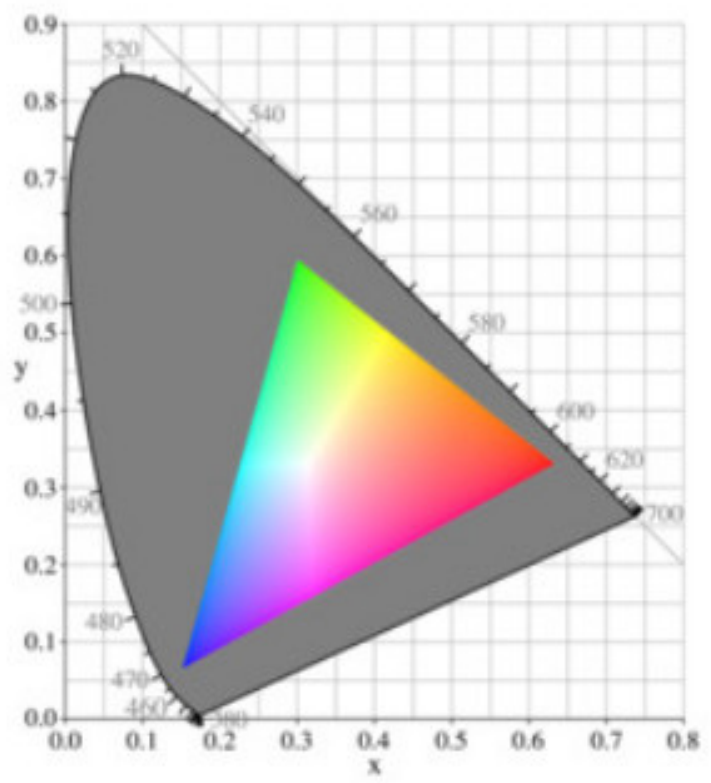

Figure 4: The colour gamut of a CRT computer monitor projected onto a 1931 CIE chromaticity diagram.

As an alternative to the abovementioned visualization (on a chromaticity diagram), $R G B$ space can also be pictured as a unit-cube rectangular Cartesian space of three perpendicular axes (shown in Figure 5). Each axis represents the excitation of one of the (Red, Green, or Blue) phosphors of the monitor (measured from the voltage supplied to the video gun of each phosphor) and thus also the contribution of that primary to a colour point within the space $24,25,29,30$. Conventionally the three axes are normalized to range from 0 (no contribution) at the origin to 1 (fully saturated) at their respective maximums, and any colour within the space can therefore be specified by those three values, usually expressed as three entries of a vector $(r, g, b)$. The diagonal line running from $(0,0,0)$ to $(1,1,1)$ is the achromatic axis or line of greys $4,14,29,30$.

Although $R G B$ space is the foundation of so much common visual technology, it is not a model that is commonly used by other industries to specify colours. This is because $R G B$ space is not very visually uniform and is considered unintuitive to use (it is difficult for an observer to select a specific colour using a $R G B$ vector without software or a transform-inter- face to assist them $\left.{ }^{4}, 14\right)$.

a)

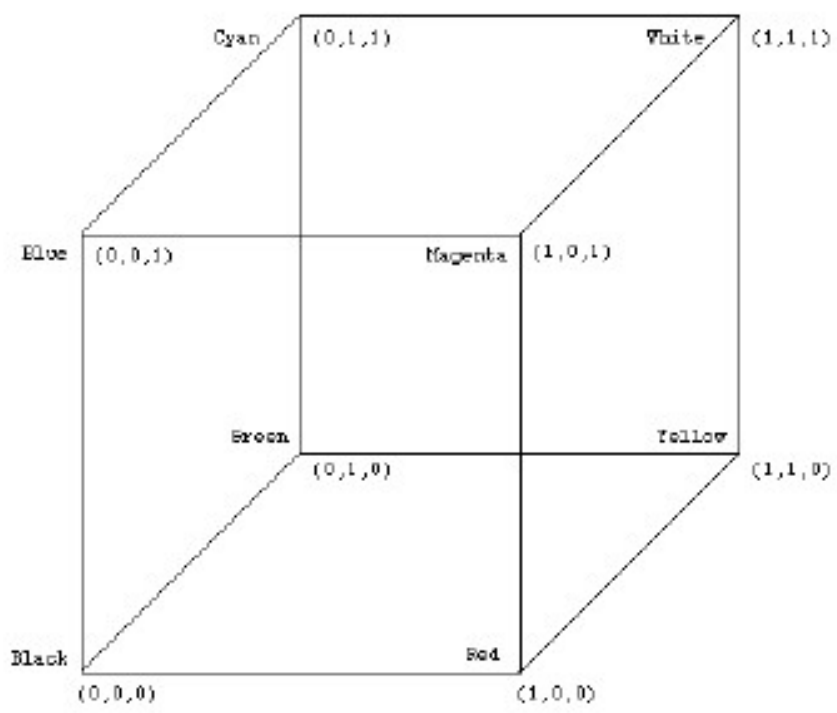

b)

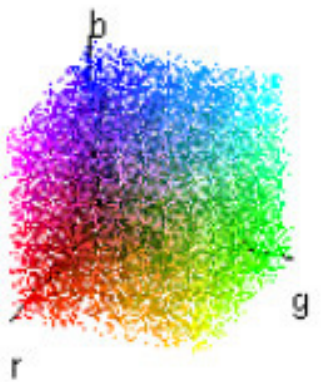

Figure 5: a) A simplified view of a $R G B$ colour cube and, b) The $R G B$ cube shown in colour from a different perspective.

\section{Derivatives of $R G B$ Space}

There are many derivatives and deformations of $R G B$ space (in addition to the many subtle variations that exist due to device-dependence), which have become popular and have been recognized as independent colour spaces. For example, HSV (Hue, Saturation and Value) colour space ${ }^{30}$ was defined by Smith in 1978 as a more artistically intuitive and potentially more user-friendly version of the $R G B$ colour cube. The space is visualized as a projection view of the $R G B$ cube along the achromatic diagonal axis in the direction from white to black. As can be seen in Figure 6 , this results in a projected (2-D) hexagon with grey at the centre and the six corners in clockwise order being fully-saturated blue, cyan, green, yellow, red, and magenta respectively. Hue, Saturation, and Value have purposefully been defined in terms that 
are not specific to colourimetry so that they are easily understood by lay-users, and collectively attempt to explain the common artistic concepts of hue, tint, shade, and tone ${ }^{30}$ : Hue refers to the basic colour and is defined as the angle around the colour hexagon from a reference to the specific colour. Saturation is considered as the departure of the colour from white and is defined as the length of the vector (on the hexagonal projection) from the central grey point to the specific colour. Finally, Value is referred to as the departure of the colour from black (the zero-energy colour) and is effectively the position where the hexagonal plane projection intersects the grey axis ${ }^{30}$. Variations of $H S V$ colour space have been the foundation of many popular computer graphics programs, such as Paint Shop Pro and Photoshop, because they allow a user to specify a desired colour much easier than any of the CIE spaces or the $R G B$ colour cube ${ }^{4,31-33}$. However, because $H S V$ space is essentially a transformation of the $R G B$ colour cube it shares all of its shortcomings, such as device-dependence and visual non-uniformity $4,31-33$.

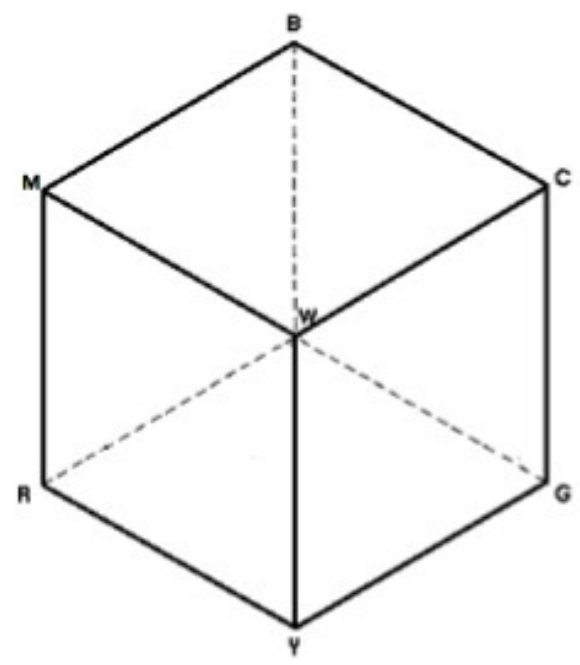

Figure 6: Hexagonal projection of $H S V$ colour space. (Adapted from $\mathrm{Smith}^{30}$ ) The solid lines show the $R G B$ cube in the orientation needed to visualize the $H S V$ space.

Some of the many variations of $H S V$ space are commonly referred to as HSI (Hue, Saturation and Intensity) and $\mathrm{HCI}$ (Hue, Chroma and Intensity). Although HSL (Hue, Saturation and Lightness) is often used interchangeably with $H S V$ in many texts, it was originally used by $\mathrm{Smith}^{30}$ to describe another (distinct) colour space. Hue and Saturation are defined as for $H S V$, but Lightness quantifies the energy in a colour rather than its non-blackness. Smith defines and vis- ualizes the HSL colour space quite differently from $H S V$ : $H S L$ is limited to the triangular plane of colours within the $R G B$ colour cube where $R+G+B=1$. The $H S L$ space is as close as one can come to an isoluminant plane in a $R G B$ colour system, and since it is a more generalized version of the $H S V$ space it has been used commonly in colour television signal programming ${ }^{30,33}$.

Many companies, such as Kodak, Xerox, and Adobe have developed other colour spaces for specific applications, which are linear transformations and quantizations of $R G B$ colour space ${ }^{4,30-32}$ and do not need to be specifically elaborated on here. The European PAL television standard ( $Y U V$ colour space) and the American NTSC (National Television Standards Committee) system (YIQ colour space) are also transformations of $R G B$ colour space , $^{4,30-32}$ and therefore will similarly not be included in further detail here.

$R G B$ space and its above derivatives are known as additive colour spaces in that they are designed for light-emitting sources (and that the colour perceived by the eye is a compound mixture of its primary colours). Another interesting group of colour spaces derived from $R G B$ space are those designed for reflective colour sources and known as subtractive colour spaces. These colour spaces were originally called $C M Y$ spaces because of their Cyan, Magenta, and Yellow primaries, but more recent versions adopted the acronym $C M Y K$ due to the addition of a black component to the systems ${ }^{32-34}$. Although, like $R G B$, a multitude of device-specific $C M Y K$ spaces exist, they can be broadly divided into two main groups: The first group is known as one minus $R G B$, and their primaries are calculated as $C=1-R ; M=1-G$; $Y=1-B$. Although being mathematically trivial in their transformation from $R G B$, they are seldom used due to their poor specification of colours ${ }^{32-34}$. Many improved transformations have been developed for specific applications in industries, such as printing, textiles, and colour photography, which use complex mathematics and look-up tables (LUT) to compensate for any overlap in the absorptive quality of the pigments or dyes used in the respective fields ${ }^{32-34}$.

\section{Cohen's Fundamental Colour Space}

Stemming from the study of metamers (colours that are perceived as identical despite having different spectral compositions; thus, visual-equivalence class- 
es of colours $1,5,7,21)$ initiated by Wyszecki, Cohen $35-37$ developed an alternative theory to the perception of colour. Briefly stated, this theory considers that the spectral representation of any colour can be divided into two vector parts: the first, called the fundamental, is the part of the colour of which the visual system is aware and is able to process, and the second, known as the residual is the part that the visual system either suppresses or is unable to process. Thus two metamers possess the same fundamental part (and are consequently perceived as identical) while their residual parts are different and account for the difference in their spectral compositions.

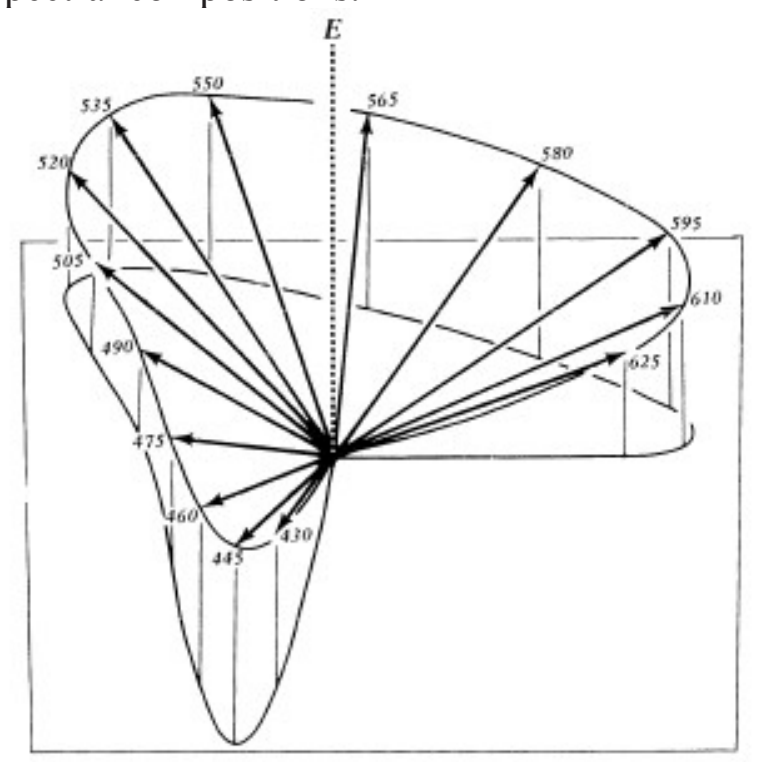

Figure 7: Cohen's fundamental colour space (from Cohen ${ }^{37}$ ) showing the fundamental vectors of monochromatic stimuli in $15 \mathrm{~nm}$ passbands from $430 \mathrm{~nm}$ to $625 \mathrm{~nm}$. From: American Journal of Psychology, Copyright 1985 by the Board of Trustees of the University of Illinois. Used with permission of the University of Illinois Press.

Cohen represented spectral curves as column matrices (vectors), and developed an operator, that he termed matrix $\mathbf{R}$, to perform the separation of the spectral vector (by matrix multiplication) into the fundamental and residual (vector) parts ${ }^{35-37}$. The dimension of these vectors depends on the size of the intervals, or passbands, used to gauge (divide-up) the spectrum. Cohen chose to use monochromatic stimuli spaced $15 \mathrm{~nm}$ apart and consequently used vectors of dimension between 14 and 21, but this was merely an arbitrary and convenient choice. (Generally, the number of passbands can vary from a relatively low number, like Cohen used, to sometimes as many as 300 , depending on how thoroughly one wishes to sample the (infinitely-dimensional) spectrum ${ }^{35-37}$.)

The 3-D colour space that Cohen constructed (shown in Figure 7) is formed by plotting the fundamental vectors of monochromatic stimuli across the visible spectrum on a 3-D Cartesian plane. All monochromatic stimuli lie on the edge of one of the "wings" of the space and all heterochromic stimuli lie within the configuration's bounds ${ }^{35-37}$. Cohen's space is an effective method of including data from the spectral distribution curves of colours into a colour space and has made it possible to associate a unique spectrum (the fundamental) with a colour (this was not possible before his work), but its use is currently limited to only a few researchers in colour science.

\section{The Perspective of Koenderink}

More recently, Koenderink has published a book ${ }^{21}$ that adds a more formal mathematic and geometric perspective to colour and colourimetry. He considers many viewpoints on colour and begins by defining an infinitely-dimensional space, the space of all beams of electromagnetic radiation between approximately 350 and $800 \mathrm{~nm}$, of which the space of colours is a 3-D subspace.

Each point in the space of beams can be mapped, using the colour matching equations (in matrix form) mentioned above, to a point in the 3-D colour space. In this way a black point is defined in colour space and all metamers of a specific colour are mapped onto the same specific point in the space ${ }^{21}$. In this definition, Cohen's fundamental space is also a 3-D subspace of the space of beams and the residual (black) space an infinity-minus-three-dimensional subspace that completely describes the colour-discrimination ability of a theoretical (standard) observer ${ }^{21}$.

The 3-D colour space can be visualized as a solid cone with its apex at the black beam. While monochromatic beams form most of the outer surface of the cone, a sector of non-spectral purples is, again, needed to close the edge of the cone, and all other (non-spectral) colours (formed by linear additions of colours on the surface of the cone) fill the inside of the cone ${ }^{21}$.

To formally qualify as a complete linear space, the space of colours needs to fulfill a few standard requirements: addition of elements (beams), scalar multiplication of elements, and the existence of a null 
element (the black, or empty, beam) are all readily satisfied, however, the additive inverse of any beam also needs to exist ${ }^{21,38}$. In other words, for any colour (beam) there needs to exist another colour that can be added to it to form a resultant black beam. This is clearly a challenge in the traditional view of colour mixing because it is never expected that when mixing two coloured lights, for instance, that one will extinguish the other and produce a black result. To overcome this problem Koenderink proposes considering ordered pairs of colours (and the difference between the pair of colors) - rather than individual colours - as the basic elements of colour space and colourimetry ${ }^{21}$.

This is not actually an unfamiliar idea: evaluating the difference between colours is a task not unlike that used to determine the colour matching functions and is analogous to the ideas of having negative contributions to a colour mixture and having virtual primary colours (both mentioned above). Although this thinking often involves the use of virtual beams, it allows colour space to be considered as a true linear vector space and agrees, in fact, with the importance of colours in optometric colour testing (simply, whether or not a difference can be distinguished between two colours).

\section{Conclusion}

\section{Usefulness of Colour Spaces in Optometry}

Optometry's involvement in colour science has traditionally concerned the detection and, sometimes, the quantification of colour vision defects. Although several effective tests for colour vision defects (such as some pseudoisochromatic plate tests) were created before advanced colourmetric measurements were available 5 , the development of colour spaces and more accurate colourimetry has allowed great advances in colour vision testing to be made, such as the computerization of colour vision assessment ${ }^{8}, 10,24,39-42$.

Colour spaces have enabled the bounds of human colour discrimination to be determined and visualized (through MacAdam ellipses ${ }^{5,42-44}$, illustrated in Figure 8), and have allowed the type and degree of a colour vision defect or deficiency to be more accurately quantified (with directional dichromat-confusion-lines, for instance ${ }^{5,42-44}$ ). By using colour spaces, the difference (or similarity) between colours can now, not only be illustrated, but can also be measured and better understood. The notion of a just noticeable (colour) difference (JND), for instance, has been refined through the development of colour spaces (all attempts at uniform colour spaces imply a JND, or a multiple thereof, as the metric (unit) used to arrange colours) and is critical to quality-control processes in the many abovementioned industries that make use of colour 45,46 .
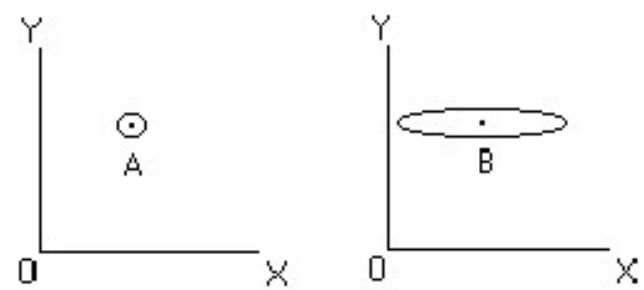

Figure 8: A simplified illustration of MacAdam ellipses. The circle (in the image on the left) and the ellipse (in the image on the right) contain all of the colour-points that cannot be distinguished from the point in the centre, by two subjects, A and B, respectively. Subject A is able to discriminate changes in colour quite evenly in both the $\mathrm{X}$ and $\mathrm{Y}$ directions (hence a circular MacAdam "ellipse"), while subject B has poorer discrimination ability in the $\mathrm{X}$ direction than the $\mathrm{Y}$ direction (hence the elongation of the ellipse in the $\mathrm{X}$ direction). When such ellipses are plotted on a chromaticity diagram (and not on the simplified Cartesian plane used here) the orientation of their elongation has been identified to correspond with specific colour deficiencies. Furthermore, when these ellipses elongate across the entire chromaticity diagram, they form the sets of (directional) dichromatconfusion-lines mentioned in the text.

From every discovery and advancement that is made in the development of colour spaces and in colour science generally, optometry benefits enormously. If the profession hopes to reap these benefits fully and possibly to contribute towards their further improvement, the deeper and more current our knowledge and understanding of these concepts concerning colour spaces will need to become.

\section{Acknowledgements}

The authors wish to thank Prof G Hoffmann for the use of Figure 3, and the Board of Trustees of the University of Illinois for the use of Figure 7.

\section{References}

1. Malacara D. Color Vision and Colorimetry: Theory and Applications. Washington: SPIE Press, 2002 1-20, 31-116. 
2. Grenfell J. Into Battle. Poem In: The Penguin Book of First World War Poetry, Ed: J. Silkin, Penguin Book Ltd, Harmondsworth, England 1996.

3. Kuehni RG. Color space and its divisions. Color Research and Application 2001 26(3) 209-222.

4. Ford A, Roberts A. Colour Space Conversions. Technical Report. University of Westminster, London August 1998.

5. Lakowski R. Theory and practice of colour vision testing. British Journal of Industrial Medicine 196926 173-189, 265-288.

6. Sharma G, Trussel HJ. Digital color imaging. IEEE Transactions on Image Processing 1997 6(7) 901-932.

7. Benjamin WJ, Borish IM. Borish's Clinical Refraction, 4th Ed. Philadelphia: W.B. Saunders, 1998.

8. Toufeeq A. Specifying colours for colour vision testing using computer graphics. Eye 200418 1001-1005.

9. Shaw M, Fairchild M. Evaluating the 1931 CIE colormatching functions. Color Research and Application 2002 27(5) 316-329.

10. Meyer GW, Greenberg DP. Color-defective vision and computer graphics displays. IEEE Computer Graphics and Applications 1988 September 28-40.

11. Judd DB. Fundamental studies of colour vision from 1860 to 1960. Proceedings of the National Academy of Sciences of the USA 1966 55(6) 1313-1330.

12. Broadbent AD. Calculation from the original experimental data of the CIE 1931 RGB standard observer spectral chromaticity co-ordinates and colour matching functions. Centre for Imaging Science, Munsell Color Science Laboratory. http://www.cis.rit.edu/mcsl/research/broadbent/CIE1931_ RGB.pdf. Date accessed: 20/12/2010.

13. Hurvich LM, Jameson D. An opponent-process theory of colour vision. Psychological Review 1957 64(6) 384-404.

14. Bourke P. RGB Colour Space. http://paulbourke.net/texture_colour/colourspace/ 1995 Date accessed 12/05/2011.

15. Feitosa-Santana C, Bimler DL, Paramei GV, Oiwa NN, Barboni MTS, Costa MF, Silveira LCL, Ventura DF. Colorspace distortions following long-term occupational exposure to mercury vapor. Ophthalmic and Physiological Optics 201030 724-730.

16. Moroney N, Fairchild MD, Hunt RWG, Li C, Luo MR, Newman T. The CIECAM02 color appearance model. IS\&T/SID Tenth Color Imaging Conference, Scottsdale, Arizona: The Society for Imaging Science and Technology.

17. Vingrys AJ, King-Smith PE. A quantitative scoring technique for panel tests of color vision. Investigative Ophthalmology and Visual Science 1988 29(1) 50-63.

18. Hoffmann G. CIE Color Space. http://fho-emden.de/ hoffmann/ ciexyz29082000.pdf. 2010 Date accessed 10/01/2011.

efg's Computer Lab. CIE Chromaticy Diagram. http://www.

19. efg2.com/Lab/Graphics/Colors/Chromaticity.htm.2010 Date accessed 10/01/2011.

20. Yendrikhovskij SN. A computational model of colour categorization. Color Research and Application 2001 Supplement 26 (s235-s238).

21. Koenderink J. Color for the Sciences. The MIT Press: Cambridge, Massachusetts, 2010.
22. Hoffmann G. CIELab Color Space. http://www.fho-emden.de/ hoffmann/cielab03022003.pdf. 2009 Date accessed 10/01/2011.

23. Hunterlab. Insite on Color. Applications Note 13(2) http:// www.hunterlab.com/appnotes/an02_01.pdf. 2008 Date accessed 10/01/2011.

24. Hoffmann A, Menozzi M. Applying the Ishihara test to a PC-based screening system. Displays 199820 39-47.

25. Derefeldt G, Hedin CE. Visualization of VDU colours by means of the CIELuv colour space. Displays 1989 10(3) 134-146.

26. Samu K, Wenzel K. Presenting surface colours on computer controlled CRT displays. Facta Universitas Series: Electronics and Energetics 2003 16(2) 117-183.

27. Cowan WB. An inexpensive scheme for calibration of a colour monitor in terms of CIE standard coordinates. Computer Graphics 1983 17(3) 315-321.

28. Meyer GW, Greenberg DP. Perceptual colour spaces for computer graphics. Computer Graphics 198014 254-261.

29. Gonzalez RC, Woods RE, Eddins SL. Digital Imaging Processing using MATLAB. New Jersey: Pearson Prentice Hall, 2004 194-241.

30. Smith AR. Color gamut transform pairs. Computer Graphics 1978 12(3) 12-19.

31. Cardani D. Adventures in HSV Space. http://visl.technion. ac.il/labs/anat/hsvspace.pdf Date accessed 21/09/2011.

32. Tkalcic M, Tasic JF. Colour spaces - perceptual, historical and applicational background. Eurocon 2003. Computer as a Tool 1 304-308. [Conference proceedings.]

33. Ebner M. Color Constancy. West Sussex, England: John Wiley \& Sons Ltd. 2007 87-103.

34. Poynton C. A Guided Tour of Color Space. http://www. poynton.com/PDFs/Guided_tour.pdf. 1997 Date accessed 23/09/2011.

35. Cohen JB. Visual Color and Color Mixture: The Fundamental Color Space. Urbana and Chicago: University of Illinois Press, 2000.

36. Cohen JB, Kappauf WE. Metameric color stimuli, fundamental metamers, and Wyszecki's metameric blacks. The American Journal of Psychology 1982 95(4) 537-564.

37. Cohen JB, Kappauf WE. Color mixture and fundamental metamers: theory, algebra, geometry, application. The American Journal of Psychology 1985 98(2) 171-259.

38. Kolman B, Hill DR. Introductory Linear Algebra - An Applied First Course. 8th Edition. Pearson Prentice Hall, 2005 272-290.

39. Mollon JD, Regan BC. Cambridge Colour Test Handbook, Version 1.1. London: Cambridge Research Systems, 2000.

40. Arden G, Gundoz K, Perry S. Colour vision testing with a computer graphics system: preliminary results. Documenta Ophthalmologica 198869 167-174.

41. Yang S, Ro YM, Wong EK, Lee JH. Quantification and standardized description of color vision deficiency caused by anomalous trichromats - Part 1: Simulation and measurement. EURASIP Journal on Image and Video Processing 2008 1-9.

42. Regan BC, Reffin JP, Mollon JD. Luminance noise and the rapid determination of discrimination ellipses in colour de- 
ficiency. Vision Research 1994 34(10) 1279-1299.

43. Simunovic MP, Votruba M, Regan BC, Mollon JD. Colour discrimination ellipses in patients with dominent optic atrophy. Vision Research 38 3413-3419.

44. Ventura DF, Simoes AL, Tomaza S, Costa MF, Lagoa M, Costa MTV, Canto-Pereira LHM, de Souza JM, Faria MAM, Silveira LCL. Colour vision and contrast sensitivity losses of mercury intoxicated industry workers in Brazil. Environmental Toxicology and Pharmacology 200519523 529.

45. Luo MR, Cui G, Rigg B. The development of the CIE 2000 color-difference formula: CIEDE2000. Color Research and Application 2001 26(5) 340-350.

46. Whitehead LA, Mossman MA. A Monte Carlo method for assessing color rendering quality with possible application to color rendering standards. Color Research and Applica-

27. tion 2012 37(1) 13-22.

Biochem Biophys Res Commun 1999265 322-325.

Glasgow BJ, Abduragimov AR, Farahbakhsh ZT, Faull KF,

28. Hubbell WL, Tear lipocalins bind a broad array of lipid ligands. Curr Eye Res 199514 363-372.

Schoenwald RD, Vidvauns S, Wurster DE, Barfknecht CF. 